\title{
Measuring Surface Atom Bond Length Contraction in Au and Pt Nanoparticles Using High-Precision STEM Imaging
}

A. B. Yankovich ${ }^{1}$, B. Berkels ${ }^{2}$, W. Dahmen ${ }^{4}$, R. Sharpley ${ }^{3}$, and P. Binev ${ }^{3}$, and P. M. Voyles ${ }^{1}$

1. Dept. of Materials Science and Engineering, University of Wisconsin, Madison, WI 53706, USA.

2. Institut für Numerische Simulation, Rheinische Friedrich-Wilhelms-Universität, 53115 Bonn, Germany.

3. Interdisciplinary Mathematics Institute, University of South Carolina, Columbia, SC 29208.

4. Institut für Geometrie und Praktische Mathematik, RWTH Aachen, 52056 Aachen, Germany.

Precision smaller than the resolution is routinely attainable in both TEM and STEM images. The best reported precision using TEM is $1 \mathrm{pm}$ [1], while for STEM it is $5 \mathrm{pm}$ [2] because STEM encounters practical limits before reaching the fundamental precision limit set by the signal to noise ratio. These practical limits include the serial acquisition nature of STEM and the smaller portion of the electron scattering collected during STEM compared to TEM. To achieve the best precision, both TEM and STEM combine multiple exposures to increase signal to noise ratio and decrease the effects of instabilities. We have developed a non-rigid (NR) registration scheme for series of STEM images that accounts for all types of image distortions caused by instabilities during acquisition [3].

Figure 1 shows the results of NR registration of a series of HAADF STEM images of GaN along $[11 \overline{2} 0]$. The images were acquired on a probe-corrected FEI Titan at $200 \mathrm{kV}$ with a $24.5 \mathrm{mrad}$ convergence angle, a $25 \mathrm{pA}$ probe, and collection angles from 54 to $270 \mathrm{mrad}$. The series consists of $512,256 \times 256$ pixel images at a $13 \mu$ s per pixel dwell time and $10.6 \mathrm{pm} /$ pixel sampling. Figure 1 (a) is the first frame of the series. Figure 1(b) shows the pixel-wise average of the NR registered frames with no smoothing or other image processing. There is no loss of spatial resolution or sampling from the NR registration even though the effective dwell time per pixel in Figure 1(b) is $6.66 \mathrm{~ms}$. We fit each of the Ga columns to a 2D Gaussian function to get sub-pixel accuracy for the atomic column positions, then calculated the interatomic distances in the $x$ and $y$ directions defined in Figure 1(b). The histograms of interatomic distances are shown in Figure 1(c) and (d), and the standard deviation of the measured interatomic distances is the precision. By this measure, the precision in the 48 measured $x$ distances is $0.74 \mathrm{pm}$, and the precision in the 42 measured $y$ distances is $0.85 \mathrm{pm}$.

Pt and Au nanoparticles (NPs) show great promise for various catalytic reactions [4]. Understanding and controlling NP surface structure is crucial in determining their chemical activity [5]. Multiple techniques, including X-ray diffraction, low energy electron diffraction, and atom beam scattering show pm-scale bond length contraction in the first few surface layers of two-dimensional metal samples. However, these techniques measure average lattice spacing and lack the spatial resolution necessary to measure bond length contractions of specific atom sites. Our NR registration technique combined with atomic resolution HAADF STEM has the spatial resolution and image precision required to measure surface atom bond length contractions in catalyst NPs that are vital to their performance as catalysts.

Figure 2 shows the results of NR registration of a series of HAADF STEM images of a Au nanoparticle on an a-C support along [110]. The series consists of $240,256 \times 256$ pixel images at a $5 \mu$ s per pixel dwell time and $60.2 \mathrm{pm} /$ pixel sampling. Figure 1(a) is from the first frame of the series. Figure 1(b) is 
the averaged image after the $\mathrm{NR}$ registration. Each $\mathrm{Au}$ atom near the nanoparticle center has an integrated scattered signal of $1.3 \times 10^{6}$ electrons. The fit positions of each column are shown by the red markers in Figure 2(b) and (c). The blue markers in Figure 2(c) are a crystallographic lattice defined on the interior of the particle and continued to the surface, so the difference between the blue and red markers signify atom displacement. Atoms more than two atomic layers from the surface show no displacement from the constructed lattice. Atoms near the surfaces show pmscale bond length contraction when compared to the lattice, and atoms near the edges of surface planes with reduced coordination and smaller number of bonds show larger contractions. The atom
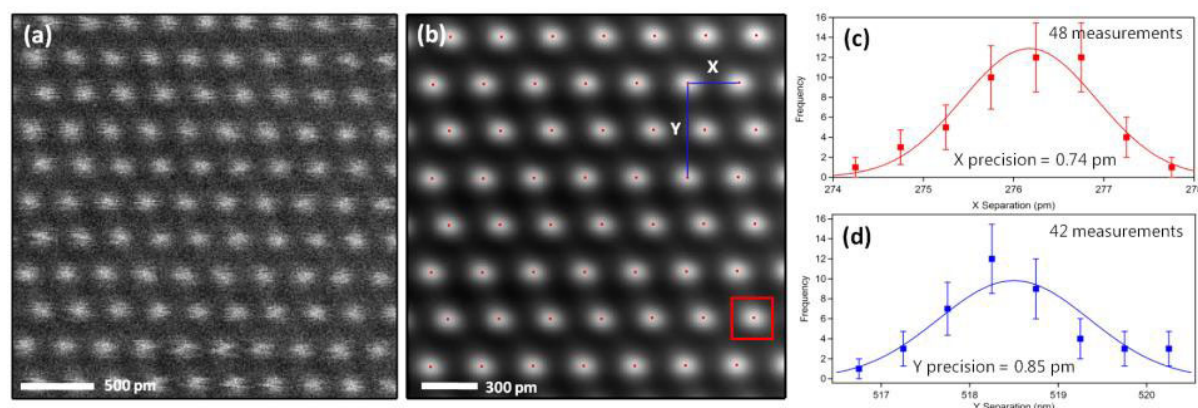

Figure 1: (a) The first of 512 frames of GaN [11-20]; (b) Average of 512 frames after NR alignment. The red dots are the positions of the columns identified by fitting. (c) and (d) The histograms of the X and Y separation measurements from (b).
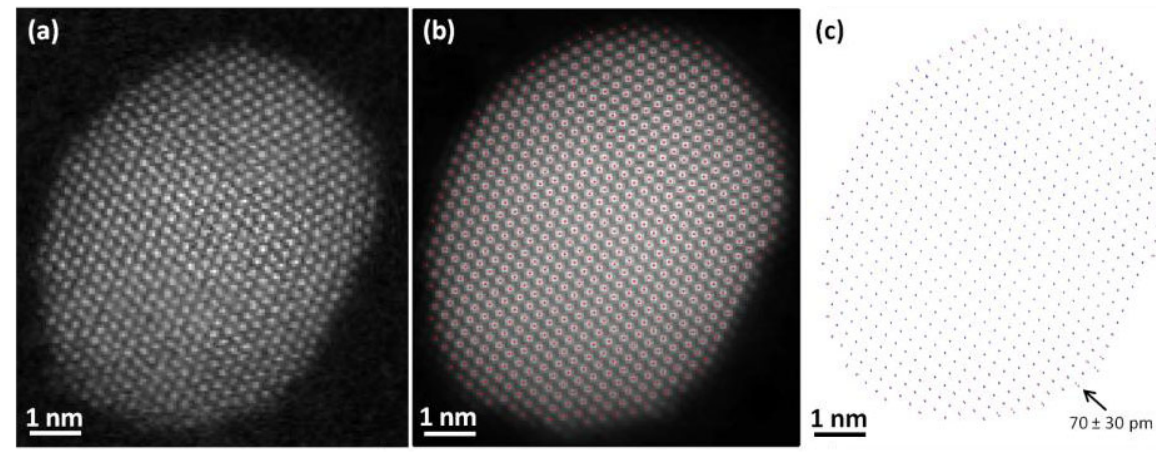

Figure 2: (a) The first of 240 frames of a Au nanoparticle along [110]; (b) Average of 240 frames after NR alignment. The red markers in (b) and (c) are the positions of the columns identified by fitting. Blue markers in (c) mark the positions of atom columns if no atom displacement was present.

column marked with the arrow in Figure 2(c) shows a contraction of $70 \mathrm{pm}$.

Results of STEM image series NR registration to measure surface atom bond length contraction for a Pt nanoparticle on silica industrial catalyst will also be presented. Variations in bond length contraction were observed in these Pt NP catalysts based on their position in the NP and their bond coordination. In addition, we have reconstructed a 3D atomic model of the $\mathrm{Pt}$ and $\mathrm{Au}$ nanoparticles by quantitatively comparing simulated and experimental STEM images [6].

References:

[1] C. Kisielowski et al., Phil. Mag. 86, 4575, (2006)

[2] K. Kimoto et al., Ultramicroscopy 110, 778 (2010)

[3] B. Berkels et al., Microscopy and Microanalysis 18 suppl. 2, 300-301 (2012)

[4] M. Schrinner et al., Science 323, 617-620 (2009)

[5] R. Narayanan et al., Nano Letters 4, 1343-1348 (2004)

[6] Acknowledgements: Work at UW was funded by the Department of Energy, Basic Energy Sciences (DE-FG02-08ER46547). Work at USC was funded by a Department of Defense ARO Multi-University Research Initiative (W911NF-07-1-0185), Special Priority Program SPP 1324 funded by DFG, the Interdisciplinary Mathematics Institute, and the College of Arts and Sciences. 